\title{
Cryo-fracturing and cryo-planning of high-pressure frozen biological samples for in-lens cryo-SEM, using a newly designed diamond knife.
}

\author{
Paul Walther \\ Central Electron Microscopy Facility \\ University of Ulm, Germany
}

High-pressure frozen rat pancreas tissue samples were cryo-fractured and cryo-sectioned with a diamond knife in the microtome of a freeze-etching device. The bulk fracture faces and blockfaces were investigated in the frozen-hydrated state by use of a cryo-stage in an in-lens SEM. With this combination relevant biological structures can be investigated with a few nanometer resolution in a near life-like state, preventing the artifacts of conventional fixation techniques. Compared to TEM replica techniques the presented method is more versatile since no replica cleaning is necessary. Additional structures can be made visible by controlled sublimation of ice, leading to a better understanding of the three dimensional organization of organelles, such as the endoplasmic reticulum.

Rat pancreatic tissue was high pressure frozen as described by Walther and Ziegler, 2002 [1] in an EM HPM high-pressure freezer [2] manufactured and distributed by Engineering Office M. Wohlwend GmbH, Sennwald, Switzerland. The frozen tissue pieces were clamped in a special holder. After transfer to the BAF 300 freeze-etching device (Bal-Tec, Principality of Liechtenstein), the temperature of the sample was raised to $163 \mathrm{~K}$ (vacuum about $2 \times 10^{-7} \mathrm{mbar}$ ) and the samples were cryo-sectioned using a special diamond knife (GFD, Gesellschaft für Diamantprodukte, Ulm, Germany; Fig 1). The properties of these artificial diamond blades are described on the GFD homepage under www.gfd-diamond.com. The knife was mounted on the microtome of the BAF 300. The microtome can be manually controlled with a wheel on the control board, outside of the bell-char. The sectioning speed was kept as slow as possible (about $0.05 \mathrm{~mm} / \mathrm{s}$ ) to prevent smearing. After sectioning, the samples were kept in the vacuum for $60 \mathrm{~s}$, in order to allow some water to sublimate ("etching"). Then the samples were double-layer coated [3]. Platinum-carbon (3 nm) was electron beam evaporated at an angle of $30^{\circ}$ during rotation of the sample ("rotary-coating") as described in Walther and Müller, 1999 [4]. A backing layer of $5 \mathrm{~nm}$ carbon was evaporated perpendicularly. After coating, the holder with the sample on it was mounted onto the Gatan cryoholder 626 (Gatan, Inc., Pleasanton, CA, USA). Specimens were investigated at a temperature of $143 \mathrm{~K}$ in the Hitachi S-5200, in-lens field emission SEM, (Hitachi, Tokyo, Japan). The primary accelerating voltage $\left(\mathrm{V}_{\mathrm{O}}\right)$ was $10 \mathrm{kV}$. Imaging was performed by collecting the backscattered electron (BSE) signal. BSEs were directly recorded with the built in YAG-BSE detector and (indirectly) with the converted BSE signal, the so-called "composite riche image". In order to improve the signal to noise ratio the YAG-BSE image and the composite rich image were superimposed. Since frozen-hydrated samples are sensitive to the electron beam, it is important to keep the irradiation dose as low as possible.

\section{References}

[1] P. Walther and A. Ziegler J Microsc. 208 (2002), 3-10.

[2] D. Studer et al., J. Microsc. 179 (1995), 321-332.

[3] P. Walther and J. Hentschel Scanning Microsc 1989, 3, (Suppl. 3), 201-211.

[4] Walther Müller J. Microsc, 196 (1999), 279-287 

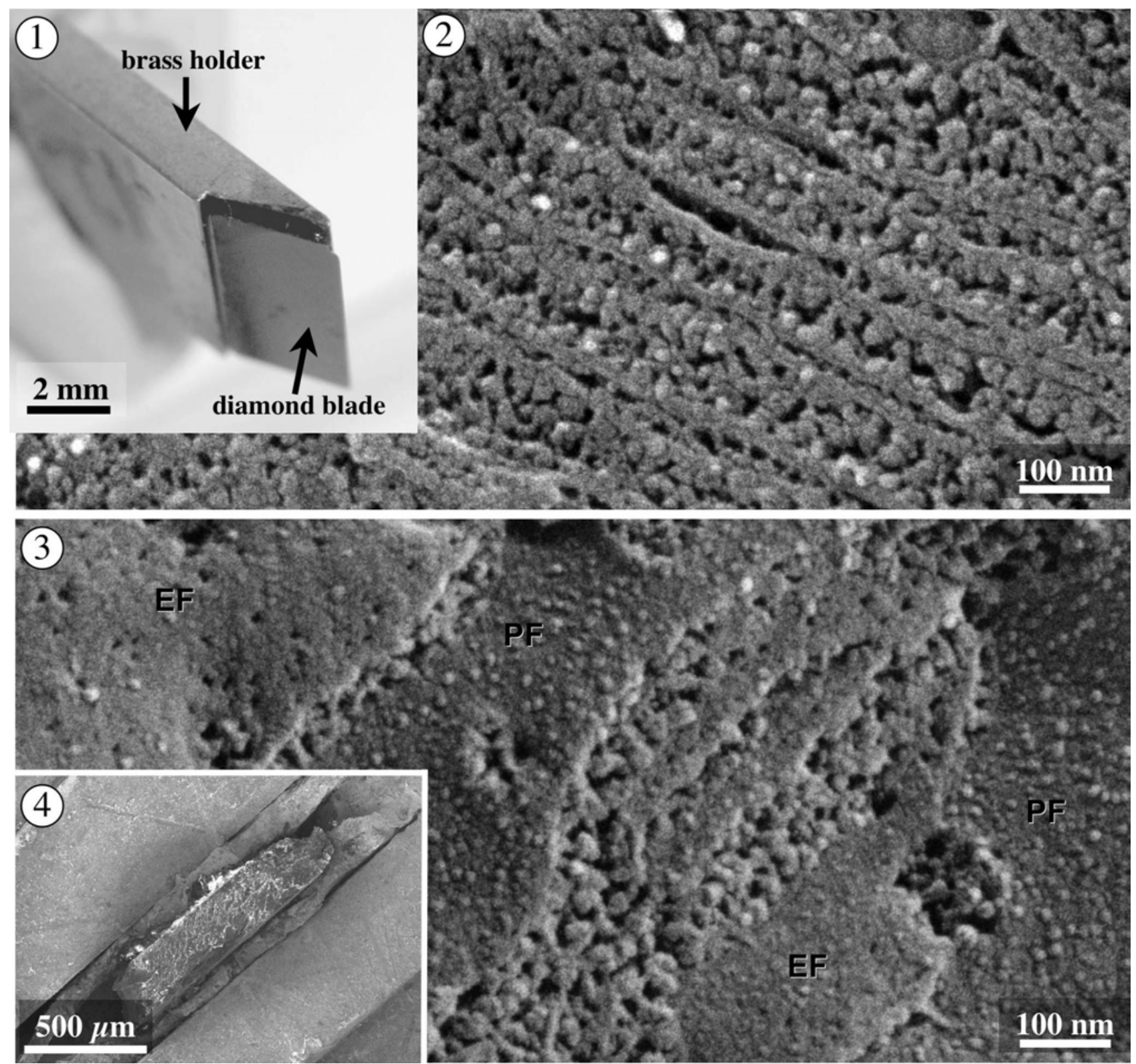

Fig. 1. The special diamond knife used in the microtome of the freeze-etching device. The diamond blade is glued onto a brass holder. Fig. 2. Perpendicular fracture to the ER membranes of pancreas tissue. Fig. 3. A more parallel fracture through a similar area, the cytosol is filled with ribosomes and some filamentous structures. The different fracture faces of the ER membranes are exposed. The smooth extraplasmic fracture face (EF) is facing the ER lumen; the rough protoplasmic fracture face $(\mathrm{PF})$ is facing the cytosol. Fig. 4. Low power image of the high pressure frozen piece of pancreatic tissue, mounted in the Bastacky holder with indium foil for good mechanical, thermal and electrical contact. 